\title{
Research on the value of search engine optimization based on Electronic Commerce
}

\author{
WANG Yaping ${ }^{1, a}$ \\ ${ }^{1}$ College of Information Science and Technology, Zhejiang shurenuniversity, Hangzhou \\ 310015, China \\ aemail: wangyaping@126.com
}

Keywords: Electronic commerce; network marketing; search engine optimization

\begin{abstract}
E-commerce sites using search engine optimization to improve web site in the search results in the index ranking, and thus get more sales opportunities. Among them, in the station optimization is the enterprise's own site architecture optimization, need to meet the search engine crawlers search habits; standing outside the optimization is search through the analysis of competitors and provide support for the development of enterprises and the decision-making.
\end{abstract}

\section{Introduction}

In recent years, with the rapid development of e-commerce, e-commerce sites springing up like mushrooms, almost all Internet technology developers pay great attention to the the improvement and promotion of the function of the website, through a variety of ways, such as improved technology development, web performance optimization, using Ajax and JavaScript framework architecture website content, in order to enhance the user experience, increase website traffic. But with the increase in the number of e-commerce sites, uneven quality, how to let more users, namely potential consumers can to the fastest speed, the most convenient way to learn the enterprise electronic commerce website promotion product information, for the enterprise to bring more benefits, has become a focus of attention to small, medium and large enterprises. Most consumers informed of the information network is through search engines, so site architecture researchers immediately thought of the of enterprise electronic commerce website search engine optimization (SEO) will be is a very good means of network marketing. Here we make a simple introduction and analysis.

After using the optimization strategy, enterprise developers to constantly sum up observation and understanding optimization effect, improved optimization methods, correct optimization errors in the process. Only in this way can make their own optimization technology will continue to rise, also can let your website ranking gradually improved. Even if their own website ranking has been on the front, and can not stop the optimization work. Because of the fierce competition in the current enterprise, other companies are entirely possible to go beyond the company's ranking by better optimization.

It is an important task to find out the information of competitors in the process of search engine optimization, and we can consider the following aspects:

One, from the search engine to start. Since the search engine optimization, the main purpose is in the same type of website ranking in front of the competitors, so the first look at the rankings of competitors, whether in the front, take a look at competitors website is not industry is already very famous site, is not a lot of news portal has been reported in the corresponding site the, to find coping and surpassing strategy.

Two, analysis of the main indicators of competitors. Usually website search engine optimization is very concerned about the index: PageRank (PR value, the influence of search engine on the web page ranking is an important factor), snapshot update, domain name, included quantity, the chain quantity and quality, classification catalogues the amount collected. If the higher the value of these indicators, then the higher the competitiveness will be. So to analyze the main indicators on their own business sites, and then determine whether the advantages of the site needs to improve the 
optimization strategy.

Three, direct access to competitors. In addition to the above influence website search engine optimization effect of indicators, but also pay attention to the user experience of the website, because search engine optimization is only a means, and the user experience is the fundamental, only good user experience can let the enterprise obtain the lasting vitality, such as fruit only to these indicators to search engine optimization is not conducive to enterprise electronic commerce development.

Four, pay attention to the traffic situation of competitors website. Estimated flow is very important a motion analysis, because companies can see from the flow in the profitability of the competitors, then again to determine their own can not be more than the other, if the flow of the opponent has a lot and I just began operations, at this time would be considered appropriate replacement keyword, after all, and a has been very successful for the website competition, the difficulty can be imagined.

Five, from the other channels to understand the reputation of competitors. Usually for vertical industry website, each industry website will belong to the industry's popular forums, and inside forum people will more or less discussed the industry of other sites, we can see others evaluation of competitors from these discussions, and from the user's point of view, analysis the advantages and disadvantages of the site, to own site optimization to bring the most direct help[1].

These five methods are very important to improve the optimization strategy, and it is very important to defeat the competitors. However, it is also important to analyze and compare with the analysis of the competitors. So the site optimization is not only to master the skills, but also to master the strategy.

Search engine optimization is a kind of popular network marketing. Enterprises through the website structure optimization and adjustment, such that the search engines can with the fastest speed included on the page, and make the enterprise important product promotion and sales page index ranking the greatest degree of front. From this point of view, the concept of search engine optimization is very intuitive, is how to make the enterprise website better rankings. Of course, part of the search engine has the so-called "PPC" search strategy, but the method for small and medium-sized enterprise cost is too high, is not realistic and belongs to human disturbance to the search engine search results, here we only from the technical level to discuss this problem. Here we can give a search engine optimization more accurate description: search engine optimization is in order to increase the rate of exposure to specific keywords to increase website visibility, and increase sales opportunities for the main purpose of a network marketing methods.

Search engine optimization is also put forward higher requirements on the electronic commerce website developer. Developers need to learn and understand the relevant knowledge of the search engine and its operating mechanism. However, it is very difficult to make a theoretical analysis of the large scale and complex structure of the search engine, and the factors that affect the ranking of the search results are very much (some search engines are as many as 100 kinds). At the same time, the well-known search engines are commercial nature, the majority of the core algorithms and operating mechanism is not easy to learn, these factors will affect the search engine optimization to a certain extent. Search engine optimization is divided into two kinds of station optimization and station optimization.

\section{Station optimization mechanism}

In the station optimization is mainly on the site structure and page storage adjustment, to adapt the search engine crawler to grab, including page optimization, tag optimization, link optimization, optimizing content and pictures optimization.

Optimization of a page: the page, to minimize the use of static[2], dynamic web page. We all know the accurate static web crawling to the search engine, which is conducive to search engine included, further that is conducive to the website keywords ranking. And dynamic pages is after by the database query to return data, search engine consumes time relatively long, alter database, which influences which affect the crawl of accuracy, apparently search engine did not like, included 
page is less also, ranking will certainly not be good. The use of iframe to copy the contents. If must be in each page repeated some of the content, or product description and not your original, you can put these contents are placed in contact border of the iframe, let the content to the user only visible of the engine, and is not visible. The brand landing page. If your site is the product customer will directly search brand products, you have to create a brand for each landing page optimization.

Two, the optimization of the label keyword should be targeted. Usually case, e-commerce website keyword can is divided into the following categories: category word for word brand, brand, brand product word, geographical terms, etc., we need reasonable for layout, select the corresponding column or page keyword optimization of these keywords. The keyword and description should be appropriate. Each page keywords and description is best not to repeat, to avoid the search engine antipathy. Home page, column page and the content page keywords should have a sense of hierarchy, the content page keywords around the column page keywords, column page keywords to key words, try not to let the search engine was is the key word in the stack. Keywords the unique web page meta tags. The page contains keywords and description meta tags, so each product page meta tags should be completely unique. Although the element content may not directly affect the ranking, unique tags will prevent duplicate content penalty. Search engine page keywords for different number is different, the home page keyword can put some more, and for the product page had better not exceed 3, because the keyword from the back to the search engine has no effect. The tag to be personalized. Let your customers can use their own products to the keyword tag, so you can start to rank the slang type of keywords, the keywords you probably never thought. Sometimes some personalized tag is widely used, so this tag can tag cloud row in front of more or more significant.

Three, the link optimization: optimization of URL. Ideally, URL should include keywords, website depth of not more than 4 pages[3], because after the 4 page basically is not indexed by search engines. In addition, the product page from the home page to reach all the better, generally 2 to 3 times click. The product page from the home page as close as possible, because the home is generally the site of PR (PageRank, is an important factor to the search engine page rank) maximum source. Many websites will be part of the product catalog on the sub classification and classification page page directory, very far away from home, from the perspective of the user or search engine optimization terms are not so ideal. In order to ensure all the station anchor text using the title attribute appropriate, to provide to the search engine on the web content enough information. Although the title attribute is not the anchor text is important, but it is also one of the factors of engine ranking. The internal text links. Search engines do not get a lot of page information from web site navigation links. But the link text is different, using keywords of the anchor text in the content page to navigation links or left column links, or links at the bottom is more valuable than, this can increase the page links to reach the number of links, so that search engine can reach the target page in a different way. The navigation links. Avoid the use of JavaScript or other multimedia navigation structure that is not conducive to spider crawling, if you have to use such a structure, at least on each page, using the normal hypertext link copy navigation.

Four, content optimization: 1 . Create a search engine optimization keywords parameter domain in the product database. As the catalog for each product are name, price and description of some of the other features, you can create a search engine optimization keywords attribute, and automatically in the page title tag, keyword tags and text in the call of the keywords. And this keyword is through the data analysis from the user search habits have to be taken out, so when a new product and add to the site, title and some other attributes will call this keyword. Because to understand the user search habits with the word is the real optimization. Product description to be unique. For many non Productizing Corporation web site, many of them are directly copied from the manufacturer's Web site, sticky content, which is very bad, at least to be rewritten content, so that it becomes pseudo original content. Because the introduction of the same product is almost identical, the search engine is very easy to distinguish from the copy, it is clear that the contents of the copy is not ranked advantage, it is not up to the effect of the optimization. Product evaluation[4]. 


\section{Stand out optimization (analysis of competitors)}

For e-commerce sites, standing outside the optimization is mainly analysis of industry site of the same general architecture and definition of the words to determine the site style has its own unique personality, so as to improve website ranking, through this process can also analyze the related information of competitors, can provide theory basis for enterprise decision.

Before start external link optimization, we usually want to analysis their competitors site advantage, because search engine optimization actually method is a kind of competition, the enterprise website optimization level high, its competitive power is high, so the company can compete on technology website, content update, site outside the chain. A full range of analysis of their own and competitors, so as to achieve rapid beyond the competitors, so that their website ranking is the most direct goal.

After using the optimization strategy, enterprise developers to constantly sum up observation and understanding optimization effect, improved optimization methods, correct optimization errors in the process. Only in this way can make their own optimization technology will continue to rise, also can let your website ranking gradually improved. Even if their own website ranking has been on the front, and can not stop the optimization work. Because of the fierce competition in the current enterprise, other companies are entirely possible to go beyond the company's ranking by better optimization.

It is an important task to find out the information of competitors in the process of search engine optimization, and we can consider the following aspects:

One, from the search engine to start. Since the search engine optimization, the main purpose is in the same type of website ranking in front of the competitors, so the first look at the rankings of competitors, whether in the front, take a look at competitors website is not industry is already very famous site, is not a lot of news portal has been reported in the corresponding site the, to find coping and surpassing strategy.

Two, analysis of the main indicators of competitors. Usually website search engine optimization is very concerned about the index: PageRank (PR value, the influence of search engine on the web page ranking is an important factor), snapshot update, domain name, included quantity, the chain quantity and quality, classification catalogues the amount collected. If the higher the value of these indicators, then the higher the competitiveness will be. So to analyze the main indicators on their own business sites, and then determine whether the advantages of the site needs to improve the optimization strategy.

Three, direct access to competitors. In addition to the above influence website search engine optimization effect of indicators, but also pay attention to the user experience of the website, because search engine optimization is only a means, and the user experience is the fundamental, only good user experience can let the enterprise obtain the lasting vitality, such as fruit only to these indicators to search engine optimization is not conducive to enterprise electronic commerce development.

Four, pay attention to the traffic situation of competitors website. Estimated flow is very important a motion analysis, because companies can see from the flow in the profitability of the competitors, then again to determine their own can not be more than the other, if the flow of the opponent has a lot and I just began operations, at this time would be considered appropriate replacement keyword, after all, and a has been very successful for the website competition, the difficulty can be imagined.

Five, from the other channels to understand the reputation of competitors. Usually for vertical industry website, each industry website will belong to the industry's popular forums, and inside forum people will more or less discussed the industry of other sites, we can see others evaluation of competitors from these discussions, and from the user's point of view, analysis the advantages and disadvantages of the site, to own site optimization to bring the most direct help[5].

These five methods are very important to improve the optimization strategy, and it is very important to defeat the competitors. However, it is also important to analyze and compare with the analysis of the competitors. So the site optimization is not only to master the skills, but also to 
master the strategy.

\section{Conclusion}

Search engine optimized by determining the keywords, optimizing page link to promote the enterprise web page ranking, and thus bring more product sales opportunities for the enterprise, is a less investment, quick electronic commerce network marketing way, for the number, the majority of small and medium-sized e-commerce enterprises is a very effective method in enterprise was established early in the product promotion and brand image establishment will play a pivotal role. Search engine optimization as a marketing tool, the final stop is designed to improve the competitiveness of enterprises in e-commerce, enterprise benefit, so we concern for the development of technology at the same time, it is also the site optimization need always adhere to a principle. In addition, for a number of full use of cheating means to carry out the key word of the "black hat" method to optimize the site is absolutely not desirable.

\section{References}

[1] Lai. Wenwen optimization of [J]. science and Technology Innovation Herald, the search engine of electronic commerce website 2011, 21: 21-21.

[2] Zhang Na. Application of SEO technology in e-commerce website [J]. small and medium enterprise management and technology, 2011, 246-246 1:.

[3] Liu Yuli, Zhao Liyan. B2B e-commerce website SEO research [J]. Hebei Industrial Science and technology, 2009, 26 (5): 458-460.

[4] Chi Ying. Enterprise website search engine optimization strategy of [J]. science and Technology Plaza, 2008, 7: 31-33.

[5] Li Liang. [J]. optimization strategy of value engineering, search engine, e-commerce sites 2011, 30 (29): 155-155. 